\title{
COVID-19 and fertility services in the United Kingdom: a biphasic qualitative study
}

\author{
B Karavadra1', A Stockl2, A H Balen ${ }^{3}$ and E P Morris4 \\ ${ }^{1}$ Norfolk \& Norwich University Hospital, Obstetrics \& Gynaecology, Colney Lane, Norwich, Norfolk, UK \\ 2University of East Anglia, Norwich Medical School, Norwich Research Park, Norwich, Norfolk, UK \\ 3The Leeds Teaching Hospital, Obstetrics \& Gynaecology, Great George Street, Leeds, UK \\ ${ }^{4}$ Norfolk \& Norwich University Hospital, Obstetrics \& Gynaecology, Colney Lane, Norwich, Norfolk, UK
}

Correspondence should be addressed to B Karavadra: b.karavadra@hotmail.co.uk

\begin{abstract}
Recently, fertility services have started resuming since COVID-19 was declared a pandemic, but there remains significant uncertainty in the way this care will be delivered in the United Kingdom. The objective of our study was to explore the impact of COVID-19 on individuals using fertility services in the United Kingdom. The study was conducted in two phases between May 2020 and July 2020: an online questionnaire involving 1212 participants and subsequent individual semistructured telephone interviews with 15 participants. Through thematic analysis, we learned from the questionnaire findings that $74 \%$ of individuals identified as White British, $21 \%$ as Black and Minority Ethnic (BAME) and $2.6 \%$ as male. Ninety-six per cent of individuals from the questionnaire explained that COVID-19 had a 'negative impact' on their fertility treatment, namely 'delay in care'. Eighty-two per cent of participants discussed concerns about the 'uncertainty' they felt about fertility services; these included the 'unknown impact of COVID-19 on pregnancy outcomes', the 'unknown impact on general gynaecology services' and the 'unknown impact of COVID-19 on fertility success'. Through semi-structured telephone interviews with 15 participants, we learned about the 'cultural pressures' individuals from BAME backgrounds faced in relation to care. Participants were mindful about the 'pressures on the service' when reopening, and therefore 'advancing maternal age', 'socio-economic background' and 'previous unsuccessful fertility treatment' were the main factors individuals considered important when 'prioritising' fertility care. Our findings can be used by fertility service providers to appreciate the patient perspective when considering the reopening of fertility services nationally and internationally.
\end{abstract}

\section{Lay summary}

The impact of COVID-19 on patients seeking or undergoing fertility treatment is not entirely known. Many patients have had their treatment postponed during the pandemic. As fertility services begin to recommence, it is important to understand how the pandemic has affected this group of patients. In addition, it is vital to appreciate and understand the patient's voice in order to ensure services take into account the patients' concerns as they begin to offer certain fertility treatments. Our study was conducted in two phases and involved an online questionnaire and individual interviews with people. We found that people were worried about services restarting and how care would be prioritised. People also discussed some of the perceived barriers to seeking fertility healthcare. Our findings highlight the importance of understanding the patient's voice when recommencing fertility services.

Key Words: - infertility $\quad$ experiences $\quad$ qualitative research $\quad$ COVID-19 and fertility

Reproduction and Fertility (2021) 2 27-34

https://raf.bioscientifica.com

https://doi.org/10.1530/RAF-20-0055
(C) 2021 The authors Published by Bioscientifica Ltd

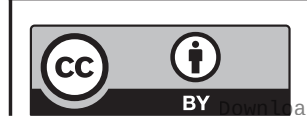

This work is licensed under a Creative Commons Attribution 4.0 International License. 


\section{Introduction}

The COVID-19 pandemic has had an unprecedented impact on the world. Fertility services in the United Kingdom were temporarily suspended when the pandemic first started. In addition, routine gynaecological workload was also stopped throughout the country. Consequently, patients who were about to undergo fertility treatment, or indeed, midway through it, had to have it stopped.

Previous public health crises including H1N1 swine flu, HIV/AIDS and the West African Ebola (Brolin Ribacke 2016) epidemic have all had a significant impact in the way healthcare is delivered, although they have not affected the provision of IVF in the United Kingdom. The COVID-19 pandemic started in Wuhan, China, in Nov/Dec 2019, with the first reported case in the United Kingdom in late January 2020 and the first deaths in the United Kingdom in March 2020. It was soon noted that measures had to be taken to create clinical capacity within acute hospitals for the pandemic and ensure that the clinical and nursing staff were appropriately prepared, trained and if necessary redeployed. The British Fertility Society (BFS) and the Association of Reproductive and Clinical Scientists (ARCS) issued guidance concerning the care of fertility patients during the pandemic, first in the form of a statement on 16 March 2020 and then a guideline on 18 March 2020. On 16 March, the ARCS and BFS made the recommendation to temporarily suspend elective assisted conception treatment (British Fertility Society). A national lockdown involving social distancing measures and restrictions in travel was announced on 23 March by the Prime Minister.

The regulatory authority of IVF clinics in the United Kingdom (the Human Fertilisation and Embryology Society, HFEA) published General Direction 0014, instructing clinics to complete active treatments by 15 April 2020. Concurrently communication from the Chief Executive and Chief Operating Officer of the NHS directed all NHS providers to, amongst other measures, prepare to 'postpone all non-urgent elective operations from 15 April at the latest, for a period of at least 3 months'.

On Friday, 1 May, the Secretary of State for Health and Social Care announced that fertility clinics would be allowed to open from May 11. The HFEA allowed clinics to apply from May 11 to resume licenced activities. A small number of clinics were able to recommence treatments shortly thereafter, with appropriate measures to reduce risk with distancing and reduced footfall through clinics. From the 15 April 2020, all non-urgent elective operations were postponed for at least 3 months (British Fertility Society).

The resumption of services has been variable and slow, as some clinical spaces were used for other purposes and many staff were redeployed to work in other parts of the health service (not only nursing and medical but also embryology and administrative). Even by August 2020 some clinics were yet to reopen.

The impact of the shutdown on service users is not exactly known and therefore this study was designed to explore this impact. The objective of the study was to explore participants' experiences of fertility care during COVID-19 and any perceived barriers to care and to gain insight into the way participants felt about fertility care upon resumption of services. The study was conducted in two phases: phase one being an online questionnaire and phase two being individual semi-structured telephone interviews.

\section{Materials and methods}

\section{Study design}

This study involved an exploratory qualitative approach through two phases. Phase one involved the use of a context-setting online questionnaire (the questionnaire is presented in Supplementary information 1, see section on supplementary materials given at the end of this article) and phase two with individual semi-structured interviews (Supplementary information 2) over the telephone. The study design, questionnaire development and semistructured interview guide were developed with the support of three clinicians, a sociologist, four patients who were waiting to undergo fertility treatment and the Chief Executive of Endometriosis UK. Consensus on the items for inclusion was reached as a team through discussion.

Through the use of various online platforms and radio broadcast messages, the questionnaire was distributed nationally. The questionnaire was initially piloted on ten participants, and feedback was obtained to make amendments as necessary (for instance, the structure of sentences and the use of terminology). In addition to demographic data, the questionnaire content included participants' experiences of fertility care in the United Kingdom, their perceptions of COVID-19 and its impact on their fertility care and perceived barriers to fertility care. Consent was obtained online by participants clicking on the questionnaire link and ticking the respective disclosure.

The findings from the questionnaire study were used to inform the second phase of the study that involved

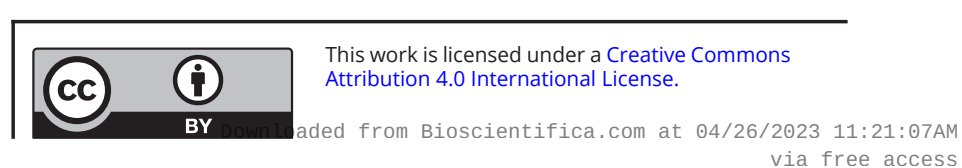


individual semi-structured interviews with participants selected through purposive sampling. Interviews are a common method of obtaining qualitative data (Whiting 2008). In addition to clinician, patient and public involvement for the design of the semi-structured interview guide, the findings from the questionnaire also guided its development. Consensus on items for inclusion was once again reached as a group. The interviews itself were conducted by $\mathrm{BK}$ and designed to further explore the findings from phase one in much greater depth. Each recorded telephone interview lasted between 30 and $108 \mathrm{~min}$. A semi-structured interview guide was used (Appendix 1). Topics identified from the online questionnaire findings for further exploration in the interviews included perceived barriers to fertility care, participants' view on the extension of embryo storage and their experiences of fertility care.

\section{Study population}

Table 1 shows the demographic details for the questionnaire respondents and semi-structured interview participants.

\section{Statistical analysis}

The findings from the questionnaire were analysed quantitatively through percentages, and the open-ended questions from the questionnaire through qualitative thematic analysis (Alhojailan 2012, Javadi \& Zarea 2016). The data were analysed separately by two researchers and through the use of a qualitative research software NVivo 1.0 (QSR International; www.qsrinternational.com/nvivoproduct). NVivo is a software that is used commonly in qualitative research to organise large amounts of qualitative data. Thematic analysis involved a number of stages including (1) familiarising with the data set (reading through the responses, making notes on an excel file), (2) generating initial codes (ascribing labels to the openended responses), (3) generating and refining themes (grouping the different codes together under common themes) and (4) relating the themes to the context of the study (the findings were related to the clinical context by the researchers) (Nowell et al. 2017).

The findings from the telephone interviews were transcribed verbatim and analysed through thematic analysis as discussed above by two researchers independently and then collaborated together. Data saturation was reached with 15 participants and this was defined as no new themes being generated from the analysis. Data saturation
Table 1 Demographic details for the questionnaire respondents ( $n=1212)$ and semi-structured interviews $(n=15)$. Data are presented as $n(\%)$.

\begin{tabular}{|c|c|c|}
\hline Demographic & Questionnaire & $\begin{array}{l}\text { Semi-structured } \\
\text { interview }\end{array}$ \\
\hline \multicolumn{3}{|l|}{ Age } \\
\hline $18-24$ & $5(0.4 \%)$ & $1(6.6 \%)$ \\
\hline $25-34$ & $889(73.3 \%)$ & $9(60 \%)$ \\
\hline $35-44$ & $316(26 \%)$ & $5(33.3 \%)$ \\
\hline $45-54$ & $2(0.16 \%)$ & 0 \\
\hline $55-64$ & 0 & 0 \\
\hline $64+$ & 0 & 0 \\
\hline \multicolumn{3}{|l|}{ Sex } \\
\hline Male & $32(2.64 \%)$ & $5(33.3 \%)$ \\
\hline Female & $1174(96.8 \%)$ & $9(60 \%)$ \\
\hline Other & $6(0.49 \%)$ & $1(6.66 \%)$ \\
\hline \multicolumn{3}{|l|}{ Geographical location } \\
\hline East of England & $182(15 \%)$ & $3(20 \%)$ \\
\hline East Midlands & $101(8.3 \%)$ & $3(20 \%)$ \\
\hline London & $133(10.9 \%)$ & $1(6.66 \%)$ \\
\hline North East & $121(9.98 \%)$ & $1(6.66 \%)$ \\
\hline North West & $98(8.08 \%)$ & $1(6.66 \%)$ \\
\hline Northern Ireland & $11(0.90 \%)$ & 0 \\
\hline Scotland & 97 (8.00\%) & $1(6.66 \%)$ \\
\hline South East & $147(12.1 \%)$ & $2(13.3 \%)$ \\
\hline South West & $133(10.9 \%)$ & 0 \\
\hline Wales & $21(1.73 \%)$ & 0 \\
\hline West Midlands & $76(6.27 \%)$ & $2(13.3 \%)$ \\
\hline $\begin{array}{l}\text { Yorkshire and } \\
\text { the Humber }\end{array}$ & $92(7.59 \%)$ & $1(6.66 \%)$ \\
\hline \multicolumn{3}{|l|}{ Ethnicity } \\
\hline White/White British & $898(74 \%)$ & $8(53.3 \%)$ \\
\hline Black/Black British & $52(4.29 \%)$ & $2(13.3 \%)$ \\
\hline Asian/Asian British & $181(14.9 \%)$ & $4(26.6 \%)$ \\
\hline Mixed race & $19(1.56 \%)$ & $1(6.66 \%)$ \\
\hline Rather not say & $1(0.08 \%)$ & 0 \\
\hline Another ethnicity & $61(5.61 \%)$ & 0 \\
\hline \multicolumn{3}{|l|}{ Highest level of education } \\
\hline $\begin{array}{l}\text { Less than secondary } \\
\text { school }\end{array}$ & $12(0.99 \%)$ & $3(20 \%)$ \\
\hline Secondary school & $321(26.4 \%)$ & $3(20 \%)$ \\
\hline $\begin{array}{l}\text { Some university, but } \\
\text { no degree }\end{array}$ & $111(9.15 \%)$ & $1(6.66 \%)$ \\
\hline Foundation degree & $31(2.55 \%)$ & 0 \\
\hline Bachelor's degree & $698(57.5 \%)$ & $7(46.6 \%)$ \\
\hline Postgraduate degree & $39(3.21 \%)$ & $1(6.66 \%)$ \\
\hline \multicolumn{3}{|l|}{ Relationship status } \\
\hline Heterosexual & $1108(91.4 \%)$ & $12(80 \%)$ \\
\hline Same sex & $66(5.44 \%)$ & $2(13.3 \%)$ \\
\hline Single person & $29(2.39 \%)$ & $1(6.66 \%)$ \\
\hline Other & $9(0.74 \%)$ & 0 \\
\hline
\end{tabular}

was defined as no new themes being generated from the data collection method (Saunders et al. 2018). Whilst 15 interviews may appear to be a small number, in qualitative research, this number generated a significant amount of data. Importantly, the inter-coder reliability between both researchers was $96.2 \%$. If there were any discrepancies, then this was discussed with a third researcher. 


\section{Ethical approval}

The study was approved by the Faculty of Medicine and Health Sciences Research Ethics Committee at the University of East Anglia. Reference: 2019/20-120.

\section{Results}

The combined findings from both aspects of the study are represented as nine themes (Table 2). The questionnaire results are discussed as percentages and these findings are further qualified by quotes from the semi-structured interviews (these are represented as brackets with the respective interview number).

One thousand four hundred and one participants clicked on the online questionnaire link, but 1212 participants replied and completed the questionnaire. Fifteen participants were recruited online to the second phase of the study involving individual semi-structured interviews.

Based on the questionnaire findings, $98 \%$ of participants used a fertility clinic in the United Kingdom within the past 12 months. Table 3 shows the most recent fertility experience and Table 4 shows the most recent type of fertility treatment that participants underwent. During the COVID-19 pandemic, the majority ( $88 \%$ of participants) were under National Health Service (NHS) fertility care and the remainder under private care.

The nine themes generated from the questionnaire findings are presented below. These include 'impact of COVID-19', 'getting pregnant during COVID-19', 'the changing nature of fertility services', 'the 10-year storage limit', 'uncertainty in treatments', 'uncertainty in service resumption', 'being diagnosed with COVID-19', 'concerns about male fertility treatment' and 'perceived barriers to accessing care'. As the semi-structured interviews were guided by the questionnaire findings, verbatim quotations

Table 2 Main themes generated from the study.

\begin{tabular}{l} 
Theme \\
\hline Impact of COVID-19 \\
Getting pregnant during COVID-19 \\
The changing nature of fertility services \\
The 10-year storage limit \\
Uncertainty in treatments \\
Uncertainty in service resumption \\
Being diagnosed with COVID-19 \\
Concerns about male fertility treatment \\
Perceived barriers to accessing care
\end{tabular}

Table 3 Fertility experience participants $(n=1212)$ had prior to fertility services temporarily stopping.

\begin{tabular}{lr}
\hline Fertility experience & $\boldsymbol{n}$ \\
\hline With a male partner & 1136 \\
With a female partner & 38 \\
No partner & 29 \\
Supporting another individual & 5 \\
Supporting another couple & 4 \\
Other & 0 \\
\hline
\end{tabular}

from the interviews were used to exemplify the main themes further.

\section{Impact of COVID-19 on patients}

Ninety-two per cent of participants from the questionnaire stated that their fertility care had been negatively impacted due to COVID-19. Reasons for this included complete cessation in treatment, not being kept up to date with when and how services may resume and uncertainty in how the virus may impact pregnancy outcomes.

\section{Getting pregnant during COVID-19}

Seventy-eight per cent of participants in the questionnaire were 'worried about getting pregnant during COVID19'. Reasons for this included concerns as to whether contracting COVID-19 would result in a miscarriage, whether patients needed to self-isolate if they were pregnant due to IVF and if COVID-19 would result in 'congenital problems' if pregnant.

It just feels so scary to get pregnant during Covid. On one hand, I want to get pregnant and have a baby, but on the other, I am scared at the thought of catching Covid and it potentially affecting the pregnancy. It makes me want to just wait until things settle. Delaying everything has really been on my mind. With Covid, is it bad that I am more worried about my potential pregnancy than myself? (Interview 14, age 21, female, White British)

Table 4 Most recent fertility treatment participants (total $n=1212$ ) underwent or had planned to undergo prior to COVID-19 lockdown.

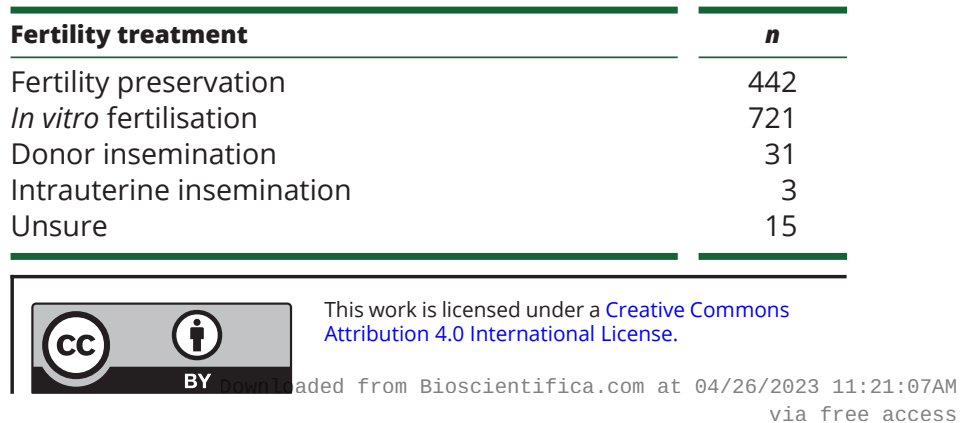


There is so much information about Covid and how it affects people. But, there just doesn't seem to be much about the way Covid can affect pregnancy and if there are any known future complications from it. My partner and I just really worry. (Interview 13, age 41, female, mixed race)

\section{The changing nature of services upon resumption}

Eighty-eight per cent of participants from the questionnaire perceived fertility care changing in the way services will be delivered once they resume again. Participants had concerns about how their care would resume once services recommenced, if clinics will be taking any extra precautions in the form of personal protective equipment and what measures services will have in place to ensure participants do not contract COVID-19. Participants also discussed concerns as to whether certain fertility services will ever resume in the way they were previously.

I know that things will have to change due to Covid. I get that. But that doesn't stop me from worrying whether I will be able to have my IVF. I just don't know how things will ever get back to normal. It just makes everything feel so hopeless. (Interview 6, age 28, female, White British)

I have read so many news articles saying that people just don't have the right sort of PPE. This worries me a lot. I am so keen to get on with my treatment, but I am also worried that I will be putting myself at risk of catching Covid from hospitals. (Interview 15, age 40, other gender, White British)

\section{Ten-year storage limit}

Participants were asked to comment on their thoughts about the 10-year storage limit on frozen embryos, eggs and sperm. In the questionnaire findings, over $80 \%$ of participants felt the additional 2-year storage limit was a positive decision, but some participants discussed their anxieties.Eight participants from the semi-structured interviews explained that an extension implied to them that future fertility care will be delayed upon resumption. One participant felt that the extension was implying that fertility services cannot cope with the patient demand and needed 'more time to help people'. Participants discussed the following:

I mean, it's great that people are allowed to have their eggs for example stored for two years longer, but the fact that the government have done this makes me anxious that there will be a delay in restarting my treatment as the extra two years may be needed. (Interview 8, age 34, female, White British)

The two-year extension is all very well, but does that mean that I will have to wait longer before I am seen again to start my treatment? (Interview 6, age 28, female, White British)

I just think that the government have introduced this new policy as they can't cope with the patient demand. More and more people want fertility care, but how can they fit everyone in? This is their way of pretending that they are helping patients, but I don't think they are really. (Interview 12, age 42, female, Black British)

\section{Uncertainties on what treatments can be offered}

Ninety-one per cent of participants from the questionnaire were concerned about what fertility treatments will be offered and to whom and when services will recommence. Participants in the interviews expanded on this:

I am worried what sort of fertility treatments my centre will be able to restart again. Even when they do restart, I wonder if they be prioritising it to certain people. There was already stigma in my community from being childless already and this has made it worse. (Interview 1, age 31, female, Asian British Indian)

Surely things will take a while to return to normal and even with the different treatments, they can't possibly get everyone in on time. There will be some criteria I bet on who gets what first. (Interview 4, age 36, male, White British)

From both the questionnaire and interview findings, participants expressed concern about the potential variation in treatments amongst fertility centres. They were also worried about how fertility services would be prioritised amongst patients. Participants discussed factors that they felt were important when deciding how services should be prioritised. These included 'advancing maternal age', 'socio-economic background' and 'previous unsuccessful fertility treatment'.

\section{Uncertainties in the way services will resume again}

Participants were worried about how centres would begin the process of restarting their treatment. During semi-structured interviews, participants discussed their anxieties about routine gynaecology care:

I am due to have a keyhole test on my fallopian tubes to check if they are working properly, but at the same time, they said I can have some endometriosis treated. I wonder 
how long this keyhole surgery will now take because of COVID. (Interview 3, age 29, female, Asian British Indian)

I have been waiting for a laparoscopy for such a long time. I guess this will never happen now. (Interview 8, age 34, female, White British)

\section{Concerns about being diagnosed with COVID-19 and its impact on fertility treatment}

Participants through both phases of the study expressed concern about being diagnosed with COVID-19. They were worried about the impact the virus would have on any developing pregnancy as well as long-term implications on a live baby. Concerns were also expressed as to whether a miscarriage was more likely or not in a COVID-positive patient.

\section{Concerns about male fertility treatments}

From both aspects of the study, participants discussed concerns about the way male fertility services may be delivered. Male participants in the interviews explained that they perceived official communication from services provides as 'female orientated' and that there was less focus on the needs of male patients.

It is great that all the official organisations are trying to keep everyone updated about fertility treatment etc, but I have found that there doesn't seem to be much focus on men who are undergoing fertility treatment specifically. I have had cancer before and so there just doesn't seem to be much information on how my fertility care might be affect or how I might be feeling. (Interview 10, age 39, male, White British)

\section{Perceived barriers to care}

Participants from a Black and Minority Ethnic (BAME) background discussed the 'cultural pressures' they faced during COVID-19 and the implications on their wider social circumstances. Through semi-structured interviews, they expressed concerns about being 'disadvantaged' from a health equalityt perspective and felt 'more at risk' from pregnancyrelated complications. Many had read about health outcomes in those individuals from a BAME background who were diagnosed with COVID-19. Participants in the semi-structured interviews expressed concerns as to whether ethnicity in general influenced fertility outcomes.

As routine gynaecology work was also suspended, participants discussed this as a barrier to fertility treatment.
Some participants were waiting for benign gynaecological surgery prior to fertility care. Other barriers included 'not being kept updated about service resumption' by some fertility centres, perceived 'lack of interest in fertility care by the government' and the influence of the media. To expand on this, participants felt that the media focussed primarily on how COVID-19-related care was delivered as opposed to fertility care. As a result of the uncertainty in the way services may be delivered, some participants considered 'postponing' their care until the pandemic was 'more stable'.

\section{Discussion}

\section{Summary of principal findings}

Through 1212 questionnaires and 15 semi-structured interviews, our findings not only provide insight into the negative impact COVID-19 has had on participants' fertility care but also highlight the professional support they have or, in the majority of cases, have not received during the closure of services. During the semi-structured interviews, we gained in-depth understanding and awareness of the barriers and concerns participants perceived to their care as services began to slowly resume particularly for those patients who were identified as male. In addition, some participants discussed how they were impacted by the cessation of routine benign gynaecological surgery. In addition, we demonstrate the absolute need for clinicians and other fertility care providers to be mindful of the changing needs of this group of patients as they may require further emotional, medical and psychological support during their treatment(s). Our findings highlight the importance of providing information that is tailored to specific concerns related to fertility and COVID-19 to patients undergoing fertility care.

During COVID-19, fertility clinics responded in different ways by alerting their patients through websites, social media, and in some cases, through personal telephone calls to keep them updated on their respective services. Furthermore, some clinical spaces were utilised for other medical functions and staff were redeployed to other clinical and non-clinical duties. Patients found this information useful as they also felt informed. Whilst a position statement released by the British Fertility Society provided insight into the resumption of fertility services in the United Kingdom, uncertainty in its exact delivery still remains. In addition, should a second wave of COVID-19 occur, there do not appear to be contingency plans in situ for the way fertility services will be delivered.

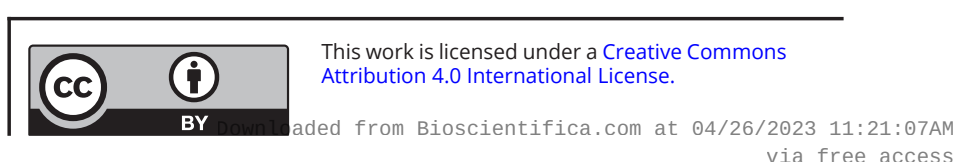


The latest WHO report on COVID-19 also does not make reference to the resumption of fertility services. As routine gynaecological surgery was also postponed during the pandemic, the government and secondary care services will need to explore how they will be able to manage this workload once services resume.

\section{Strengths and weakness of the study}

Numerous reports were released through the British Fertility Society on how fertility services can be reintroduced. However, to our knowledge, there are no other published studies in the United Kingdom involving semi-structured interviews that explored the impact of COVID-19 on individuals who are undergoing or about to undergo fertility treatment. Our study provides significant insight into the way participants perceived COVID-19 to have influenced their care and the support they received during the pandemic from their respective fertility centre. Participants also discussed the perceived barriers to care and their concerns about resuming fertility services. The findings from our study highlight the importance of the patient's voice when considering the resumption of fertility services.

Due to COVID-19 and the social restrictions imposed by the government, recruitment to the study was via online media. As a result, our findings may have captured those individuals who are social media active and therefore may under-represent the findings from those who are not. Whilst attempts were made to ensure a fair representation from individuals from a variety of demographics, the overall majority of participants were from a White British background and therefore this may have influenced the findings.

The number of replies from individuals who identified as male were also low, and as such the views of this group were under-represented. The findings from this UK study may not necessarily represent the views from those individuals throughout the world. Finally, the study was conducted during May 2020 to July 2020 and therefore the findings are representative of a time where our knowledge on COVID-19 was limited. Since this time, knowledge in this area in relation to pregnancy in particular has advanced.

\section{Findings in relation to other studies}

Our findings echo similar messages from other studies. Vaughan et al. (2020) found that during COVID-19 infertility was the main stressor that participants were concerned about. Boivin et al. (2020) provide a very interesting insight into the coping mechanisms used by fertility patients during the pandemic. Both studies demonstrate why our study is important for those providing fertility care throughout the world. Our study is unique in that it involved interviews with participants, and we were able to explore further insightful information in detail, particularly relating to barriers in accessing fertility care and how participants' perceived care should be prioritised upon resumption of services.

\section{Future research}

Our study provides the basis for further qualitative work. Undoubtedly, it may take some time before services resume back to the 'pre-COVID-19' era and therefore, services may need to be prioritised amongst patients. How this prioritisation is done is a question for stakeholders, clinicians and academics to decide with the engagement of patient and public involvement.

\section{Conclusion}

The impact of COVID-19 has been far reaching for many individuals. For those undergoing fertility care, it has been a particularly challenging and unpredictable time. This study has truly enabled participants' voices to be heard. The findings from this study can be used by fertility service providers to appreciate the patient perspective when considering the reopening of fertility services nationally and internationally and be mindful of patient concerns.

\section{Supplementary materials}

This is linked to the online version of the paper at https://doi.org/10.1530/ RAF-20-0055.

\section{Declaration of interest}

E P M has received a grant from Gedeon Richter as the UK Chief Principle Investigator for the PREMIUM study. E P M has also received consultancy fees from Chugai Pharma. E P M has been an adviser for Pfizer in 2018 and Gedeon Richter in 2019. No other conflict of interest for any of the other authors.

\section{Funding}

This research did not receive any specific grant from any funding agency in the public, commercial or not-for-profit sector.

This work is licensed under a Creative Commons Attribution 4.0 International License. 


\section{Data availability}

The data underlying this article will be shared on reasonable request to the corresponding author.

\section{Author contribution statement}

B K conceived the study, obtained ethical approval and was involved in the design, execution, analysis, manuscript drafting and critical discussion. A S was involved in the analysis, manuscript drafting and critical discussion. A $B$ and E P M were involved in the design, manuscript drafting and critical discussion. All authors approved the final version of the manuscript.

\section{Acknowledgements}

The authors thank all the participants who kindly offered their time to participate in the study and also Emma Cox from Endometriosis UK for her input with the questionnaire design and for advertising the questionnaire via their online platform. The authors are grateful for Fertility Network UK (FNUK) in advertising their questionnaire online.

\section{References}

Alholjailan MI 2012 Thematic Analysis: A critical review of its process and evaluation. West East Journal of Social Sciences $139-47$.

Boivin J, Harrison C, Mathur R, Burns G, Pericleous-Smith A \& Gameiro S 2020 Patient experiences of fertility clinic closure during the COVID-19 pandemic: appraisals, coping and emotions. Human Reproduction 35 2556-2566. (https://doi.org/10.1093/humrep/deaa218)

Brolin Ribacke KJ, van Duinen AJ, Nordenstedt H, Hoijer J, Molnes R, Froseth TW, Koroma AP, Darj E, Angel Bolkan H \& Ekström A 2016 The impact of the West Africa Ebola outbreak on obstetric health care in Sierra Leone. PLOS ONE 11 e0150080.

Coronavirus (COVID-19) guidance for patients. (available at: https:// www.hfea.gov.uk/treatments/covid-19-and-fertility-treatment/ coronavirus-covid-19-guidance-for-patients/). Accessed on June 2020.

Coronavirus (COVID-19) guidance for professionals. (available at: https:// www.hfea.gov.uk/treatments/covid-19-and-fertility-treatment/ coronavirus-covid-19-guidance-for-professionals/). Accessed on June 2020.

European Society of Gynaecological Endoscopy (ESGE) guidance. (available at: https://esge.org/wp-content/uploads/2020/04/ ESGE-Gynaecological-surgery-during-Covid-outbreak-updated-31March-2020.pdf). Accessed on June 2020.

Guidance for the care of fertility patients during the coronavirus COVID-19 pandemic. (available at: https://www. britishfertilitysociety.org.uk/2020/03/18/guidance-for-the-careof-fertility-patients-during-the-coronavirus-covid-19-pandemic/). Accessed on June 2020.

Javadi M \& Zarea K 2016 Understanding thematic analysis and its pitfalls. Journal of Client Care 1 33-39. (https://doi.org/10.15412/J. JCC.02010107)

Nowell LS, Norris JM, White DE \& Moules NJ 2017 Thematic analysis: striving to meet the trustworthiness criteria. International Journal of Qualitative Methods 16 1-13. (https://doi. org/10.1177/1609406917733847)

Position statement on the resumption of fertility treatment in the UK during COVID-19 pandemic. British Fertility Society. (available at: https://www.britishfertilitysociety.org.uk/2020/05/01/positionstatement-on-the-resumption-of-fertility-treatment-in-the-uk-duringcovid-19-pandemic/). Accessed on May 2020.

Reflections of a fertility doctor through the COVID-19 crisis. (available at: https://www.bionews.org.uk/page_149773). Accessed on June 2020.

Royal College of Obstetricians and Gynaecologists guidance on Coronavirus (COVID-19) infection in pregnancy. Version 9. (available at: https://www.rcog.org.uk/globalassets/documents/ guidelines/2020-05-13-coronavirus-covid-19-infection-in-pregnancy. pdf). Accessed on May 2020.

Saunders B, Sim J, Kingstone T, Baker S, Waterfield J, Bartlam B, Burroughs H \& Jinks C 2018 Saturation in qualitative research: exploring its conceptualization and operationalization. Quality and Quantity 52 1893-1907. (https://doi. org/10.1007/s11135-017-0574-8)

The Association of Reproductive and Clinical Scientists (ARCS) and British Fertility Society (BFS) U.K. Best practice guidelines for reintroduction of routine fertility treatments during the COVID19 pandemic. British Fertility Society. (available at: https://www. britishfertilitysociety.org.uk/2020/05/06/arcs-and-bfs-u-k-bestpractice-guidelines-for-reintroduction-of-routine-fertility-treatmentsduring-the-covid-19-pandemic/). Accessed on May 2020.

Vaughan D, Shah J, Penzias A, Domar A \& Toth T 2020 Infertility remains a top stressor despite the COVID-19 pandemic. Reproductive Biomedicine Online 41 425-427. (https://doi.org/10.1016/j. rbmo.2020.05.015)

Whiting LS 2008 Semi-structured interviews: guidance for novice researchers. Nursing Standard 22 35-40. (https://doi.org/10.7748/ ns2008.02.22.23.35.c6420)

World Health Organisation (WHO) report Coronavirus disease (COVID19) situation reports. (available at: https://www.who.int/docs/ default-source/coronaviruse/situation-reports/20200315-sitrep-55covid-19.pdf?sfvrsn=33daa5cb_6). Accessed on May 2020.

Received in final form 31 January 2021

Accepted 8 February 2021

Accepted Manuscript published online 8 February 2021 https://raf.bioscientifica.com

https://doi.org/10.1530/RAF-20-0055 (c) 2021 The authors Published by Bioscientifica Ltd

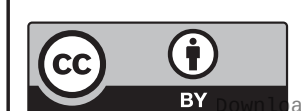

This work is licensed under a Creative Commons Attribution 4.0 International License. 